at birth. We used logistic regression models fitted with generalised estimating equations.

Results: There were 598 pregnancies in 503 women ( $32 \pm 5$ years), and 5651 pregnancies in 4086 women ( $31 \pm 5$ years) in the csDMARDs exposed and unexposed groups, respectively. The adjusted odds ratio (OR) for exposure to csDMARDs preconception and during pregnancy and risk of MCM was 1.60 (95\% $\mathrm{CI}, 1.19,2.14$ ) (table 1). In subgroup analyses, risk of MCM births was highest in those exposed to Group 2 csDMARDs during pregnancy (OR 3.63, 95\% Cl, 1.21, 10.92). For methotrexate specifically, we observed an OR of $1.62(95 \% \mathrm{Cl}, 0.53$, 4.91).

Abstract THU0697 - Table 1. Association of csDMARD exposure preconception and/or during pregnancy and risk of MCM

\begin{tabular}{|c|c|c|c|}
\hline Models* & Exposure period & OR & $\begin{array}{c}95 \% \text { Confidence } \\
\text { Interval }\end{array}$ \\
\hline Model 1 & Preconception only vs. unexposed & 1.37 & $0.84-2.24$ \\
\hline \multirow[t]{2}{*}{ All csDMARDs } & During pregnancy only vs. unexposed & 2.39 & $1.20-4.77$ \\
\hline & $\begin{array}{l}\text { Preconception AND during pregnancy vs. } \\
\text { unexposed }\end{array}$ & 1.60 & $1.19-2.14$ \\
\hline Model 2 & Preconception only vs. unexposed & 1.04 & $0.55-1.99$ \\
\hline Group 1 & During pregnancy only vs. unexposed & 2.16 & $1.02-4.61$ \\
\hline csDMARDs & $\begin{array}{l}\text { Preconception AND during pregnancy vs. } \\
\text { unexposed }\end{array}$ & 1.49 & $1.09-2.05$ \\
\hline Model 3 & Preconception only vs. unexposed & 1.43 & $0.73-2.77$ \\
\hline Group 2 & During pregnancy only vs. unexposed & 3.63 & $1.21-10.92$ \\
\hline csDMARDs & $\begin{array}{l}\text { Preconception AND during pregnancy vs. } \\
\text { unexposed }\end{array}$ & 1.71 & $1.06-2.76$ \\
\hline Model 4 & Preconception only vs. unexposed & 1.19 & $0.49-2.87$ \\
\hline Methotrexate & During pregnancy only vs. unexposed & 3.06 & $0.55-16.90$ \\
\hline only & $\begin{array}{l}\text { Preconception AND during pregnancy vs. } \\
\text { unexposed }\end{array}$ & 1.62 & $0.53-4.91$ \\
\hline
\end{tabular}

*All models were adjusted for baseline covariates including maternal characteristics, obstetrical history, comorbidities, and medication use before/during pregnancy.

Conclusions: We found associations with exposure to csDMARDs before and during pregnancy, namely the first trimester, with MCMs. These have implications for informing women with IA who are pregnant or planning to become pregnant. Disclosure of Interest: None declared

DOI: 10.1136/annrheumdis-2018-eular.4851

\section{THU0698 CLINICAL OUTCOME OF ULTRASONOGRAPHIC DETECTED UNDIFFERENTIATED SYNOVITIS IN PATIENTS WITH POLYARTHLAGIA}

M. Mortada, F.I. Abdelrahman, S. Alyan. Rheumatology, Faculty Of Medicine Zagazig University, Zagazig, Egypt

Background: Ultrasonography (US) has better sensitivity than clinical evaluation for the detection of synovitis in early inflammatory arthritis. The clinical importance and fate of undifferentiated synovitis in patients with polyarthralgia are still unknown. ${ }^{1}$

Objectives: To identify clinical outcome of undifferentiated synovitis detected by ultrasound in patients with polyarthralgia and to identify variables that can predict differentiation into specific type of arthritis.

Methods: New patients with polyarthralgia and undifferentiated synovitis were followed by clinical and ultrasound examination in the period between June 2015 to June 2017. Undifferentiated synovitis was defined as ultrasound detected synovial hypertrophy in cases in which no definitive diagnosis can be made.

Baseline assessments included: clinical examinations of complaining joints, laboratory investigations (CBC, ESR, CRP, RF, ACPA, ANA) and ultrasonographic evaluation of fifty joints (DIPs, PIPs, MCPs, wrists, elbows, shoulders, hips, knees, ankles and MTPs)

Follow ups were every $6-8$ weeks until remission of synovitis or a definite clinical diagnosis was reached. Remission was defined as absence of a previously US detected synovitis in two consecutive follow-ups eight weeks apart.

Patients were received treatment according to the opinion of the treated rheumatologist who had a full data about clinical, laboratory and ultrasonographic data. Multivariate logistic regression analysis was used to identify predictors of remission, RA, PsA and continuation as undifferentiated synovitis.

Results: 174 patients (88.6\% women, mean age $43.2 \pm 14.2$ years (range $17-75)$ ) were included.

Duration of follow up period ranged from 3 to 38 months (mean 11.4 months)

Final diagnoses were as follow; complete remission in $73(41.7 \%)$ cases, rheumatoid arthritis (RA) in 20 cases (11.4\%), psoriatic arthritis (PSA) IN 10 cases $(5.7 \%)$, spondyloarthropathy (SPA) in 11 cases $(6.1 \%)$, sarcoidosis in 4 cases $(2.3 \%)$, osteoarthritis (OA) in 10 cases $(5.7 \%)$ and 46 cases $(26.4 \%)$ continued as undifferentiated synovitis up to the end of the follow up period.
Remission of undifferentiated synovitis was associated with significantly older mean age, acute onset and absence of PD activity (OR 1.92, 1.95 and 1.86 respectively).

Development of RA was associated with chronic onset, positive rheumatoid factor, positive ACPA and US detected synovitis in more than three joints (OR 1.91, $1.75,1.92$ and 1.79 respectively). All patients with final diagnosis of RA had US detected synovitis in wrist joint with increased PD activity. PsA was associated with chronic onset, PD activity, bilateral knee synovitis, negative RF and ACPA (OR 1.84, 1.83, 1.94, 1.88 and 1.79 respectively). Cases with significantly younger mean age and shorter disease duration completed with undifferentiated synovitis (OR 1.92 and 1.87).

Conclusions: In our study, more than halve of cases of ultrasound detected undifferentiated synovitis in patients with polyarthralgia differentiated into specific type of arthritis during the follow up period. older age and acute onset of symptoms are associated with remission while positive rheumatoid factor, positive ACPA US detected synovitis in more than 3 joints including wrist joints are associated with development of RA. Bilateral knee synovitis are in favour of PsA and OA.

\section{REFERENCE:}

[1] Zufferey P, et al. Joint Bone Spine 2017 May;84(3):299-303. doi:10.1016/ j.jbspin.2016.05.011

Disclosure of Interest: None declared

DOI: 10.1136/annrheumdis-2018-eular.3525

\section{THU0699 PREDICTORS OF FRACTURE AND LOW BONE MINERAL DENSITY IN PATIENTS WITH HISTORY OF PARENTAL FRACTURE}

M. Dey, M. Bukhari. Rheumatology, University Hospitals of Morecambe Bay NHS Foundation Trust, Lancaster, UK

Background: Predictors of fragility fracture (FF) risk and low bone mineral density (BMD) in the general population are well-documented. Previous studies have shown strong familial association between parental and offspring BMD and hip fracture, varying according to factors including body mass index and corticosteroids. Little data exists on predictors of FF and BMD in patients with a family history of fracture, despite this increasing fracture risk.

Objectives: We aimed to evaluate predictors of FF and low BMD in patients attending for dual energy X-ray absorptiometry scanning.

Methods: Patients referred for BMD estimation, between 2004 and 2016, with a history of parental fracture, were included. Parameters recorded: femoral and vertebral BMD, height, weight, fat mass, age, smoking, alcohol, corticosteroids, aro matase inhibitors, Depo-Provera, hormone replacement therapy (HRT), rheumatoid arthritis (RA), polymyalgia rheumatica (PMR), breast or prostate cancer, and coeliac disease.

Logistic regression was used to model fracture risk, and linear regression was used to model the impact of each factor on vertebral and femoral BMD.

Results: 6053 patients (5513 female) were included. 2094 patients (34.6\%) had sustained at least one fracture. Smoking, alcoholism, corticosteroid, increased age, height, and fat mass significantly increased fracture risk. Coeliac disease, HRT, and aromatase inhibitors were protective. Cancer, aromatase inhibitor use, and female gender significantly decreased vertebral BMD. Corticosteroids, RA, and PMR significantly decreased L1-2 BMD. Increased age and height, and decreased weight, fat mass, and tissue thickness decreased vertebral and righ femoral BMD; this significantly increased FF risk. Corticosteroids, RA, PMR, Depo-Provera, female gender, and aromatase inhibitors decreased BMD; in the left femur, alcohol, corticosteroid use, increased age, height, and decreased weight decreased BMD

Conclusions: Similar to studies in the general population, smoking, alcohol, and corticosteroid therapy increase fracture risk, while HRT decreases it. Chronic aromatase inhibitor use increases fracture risk, suggesting a dose-dependent effect. Coeliac disease was found to be protective; previous studies have shown coeliac disease to decrease BMD, with a variable impact on fracture. Concurrent with previous studies, a differential effect of BMD in the dominant and non-dominant hip was found, with decreased right femoral BMD significant for fracture risk. Limitations of this study include lack of dose and duration of medications and lack of comparative data before and after fracture in a single patient.

Our study confirms the effect of the above factors on spinal BMD, but suggests a differential effect of smoking and alcohol on L1-2, with corticosteroids, RA and PMR affecting the lower lumbar spine. Our results also suggests a differential effect of the studied factors on the right femur compared to the left, suggesting the dominant femur is more susceptible to factors decreasing BMD. Limitations of this study include the large proportion of female subjects and lack of data on dose and duration of medications studied.

Disclosure of Interest: None declared

DOI: 10.1136/annrheumdis-2018-eular.2286 